\title{
The effect of social support and gender on depression and quality of life in patients with schizophrenia
}

\author{
Mariam Unwala ${ }^{1}$ \\ ${ }^{1}$ Post Graduate Student, Department of Psychology, Maniben Nanavati Women's College, Mumbai \\ E-mail-mariam.unwala285@gmail.com
}

\section{ABSTRACT \\ Social support buffers against stressful life events, increases} adherence to medical treatments and improves recovery from medical illness. The quality of life of patients suffering from schizophrenia is dependent on amount of social support to a great extent. Also rates of depression are high in schizophrenia patients. So we undertook this study among 68 patients to understand the relationship of social support and gender with depression and quality of life amongst the patients of schizophrenia. The scales used were the Multidimensional Scale of Perceived Social Support (MSPSS), Calgary Depression Scale for Schizophrenia and the WHO Quality of Life- Bref Scale. It is observed that means score of depression was higher in low social support group and amongst females. Similarly QOL was poorer amongst low social support groups and females. The difference was statistically significant only in $Q O L$ scores in social support group and depression scores in gender group. Thus the study concludes that social support has an impact on depression and quality of life amongst the patients of schizophrenia.

Key words: schizophrenia, social support, depression, quality of life, gender.

\section{INTRODUCTION}

Social support is defined as "Support accessible to an individual through social ties to other individuals, groups and a larger community" [1]. In general population, social support buffers against stressful life events, increases adherence to medical treatments and improves recovery from medical illness [2]. The impact of social isolation ranges from poor outcomes in terms of quality of life and self-esteem to a less favourable illness course with more psychotic symptoms or more frequent and prolonged hospitalizations [3]. Quality of Life (QOL) can be defined as an overall sense of wellbeing, comprised of both objective and subjective evaluations of physical, material, social and emotional well-being together with personal development and purposeful activity [4]. In the last two decades, there has been increasing interest in quality of life in schizophrenic patients, since schizophrenia is a severe, disabling, lifelong disorder, associated with severe social and occupational dysfunction. It has previously been shown that the quantity of supportive social relationships is predictive of subjective QOL in persons diagnosed with severe mental illness [5-6]. Especially when present within a 
context of large network size, social support can promote recovery in people with serious mental illness [7]. Nowadays, the main goal of psychiatric care is the improvement of the patients' quality of life [8]. Depression has been found as a comorbidity with schizophrenia [9]. So we took up this study to understand the relationship of social support and gender with depression and quality of life amongst the patients of schizophrenia.

\section{METHODOLOGY}

The study was conducted on 68 patients suffering from schizophrenia in various psychiatric hospitals and rehabilitation centres. The demographic details was taken in a semi-structured proforma and following scales were applied:

Multidimensional Scale of Perceived Social Support [10] : Multidimensional Scale of Perceived Social Support (MSPSS) by Zimet, Dahlem and Farley (1998), was a measure of the perceived availability of support. Twelve items assessing 3 sources of support: family, friends, and significant others. Items were rated on a 7-point Likertscale ranging from 1 (very strongly disagree) to 7 (very strongly agree).

Calgary Depression Scale for Schizophrenia [11]: Calgary Depression Scale for Schizophrenia was used for the assessment of depressive symptoms separate from positive, negative and extra pyramidal symptoms in people with schizophrenia.

WHO Quality of Life- Bref Scale [12]: The WHO QOL-BREF is an abbreviated version of the WHO QOL-100 which was developed simultaneously in 15 field centres around the world. It is a self administered instrument, however respondent who have difficulty such as illiterate can be assisted by an interviewer who reads the question. The WHO-QOL instrument, by focusing on the individuals own view of their wellbeing, provides the new perspective on the disease. It can assess the quality of life in a variety of situations and population groups. It takes into consideration physical, psychological, social relationships and environmental domains of quality of life and gives sub scores on above mentioned domains. It consists of 26 items, scored on 5 point Likert scale. The score ranges from 0 to 400 . Each domain has maximum score of 100 .

\section{Statistical Analysis}

The data was analysed using IBM SPSS version 17.0 for windows 7.

\section{RESULTS}

Total population under study was sixty-eighty participants with thirty-four males and thirty-four females. The educational qualification was better of the males as compared to the females. The males had acquired higher secondary and tertiary education ( 25 out of $34,73 \%$ ) as compared to the females ( 21 out of $34,61 \%$ ). Also moreover males came from joint families and had more support (23 out of 34 , $67 \%$ ) as compared to females (20 out of $34,58 \%$ ). For statistical analysis only the scores of low and moderate perceived social support was considered and not high scores since from the sample of sixty-eight participants, only six participants acquired high scores on perceived social support. Table 1 shows the mean values of depression and QOL scores in groups based on gender and social support. It is observed that means score of depression was higher in low social support group and amongst females. Similarly QOL was poorer amongst low social support groups and females. The difference 
was statistically significant only in QOL scores in social support group and depression scores in gender group.

Table 1 : Scores on the depression and QOL scales

\begin{tabular}{|cccc|}
\hline Groups & & Depression (Mean \pm S.D.) & QOL (Mean \pm S.D.) \\
\hline Social support & Low & $10.17 \pm 5.774$ & $190.67 \pm 42.692$ \\
\cline { 2 - 4 } & Moderate & $7.31 \pm 4.497$ & $234.12 \pm 39.334$ \\
\hline F, p value & $3.987,0.05^{*}$ & $16.129,<0.01^{*}$ \\
\hline \multirow{2}{*}{ Gender } & Male & $7.52 \pm 4.898$ & $219.23 \pm 53.46$ \\
\cline { 2 - 4 } & Female & $10.42 \pm 5.614$ & $198.55 \pm 35.85$ \\
\hline F, p value & $4.580,0.03^{*}$ & $3.624,0.06$ \\
\hline
\end{tabular}

*significant $(p<0.05)$

\section{DISCUSSION}

The purpose of our study was to understand the relationship of social support and gender with depression and quality of life amongst the patients of schizophrenia. It was observed that depression was not statistically significantly related with perceived social support but it was statistically significant according to gender, being more amongst females. It was reverse in case of QOL, i.e. statistically significant difference in terms of perceived social support seen, low social support group had poorer QOL. No difference in QOL according to gender.

This in accordance to various studies which showed that levels of social support does have an impact on the patients suffering from schizophrenia [13-15]. During the interview conducted by the researcher, while interacting with the patients, it was observed that those patients who were supported by their families, spouses, friends, neighbours and by the people who daily interacted with them, it had a positive impact. They felt that they were a part of the society and not different when they were acknowledged by these immediate people with who they met on a daily basis or once in a while. This can be corroborated by past research support that social support buffers against stressful life events, increases adherence to medical treatments and improves recovery from medical illness [2]. One of the main highlighting features of the patients suffering from schizophrenia is their level of disorientation. Many of them were not very much in touch with the reality because of their illness. Thus because of the social support that they received, it helped them to stay in touch with the reality [16-17].

Also taking into consideration that schizophrenia is a grave and a life long illness in which the patients need to undertake medications for life long. Such patients do not only go through schizophrenic symptoms but along with it they go through a depression phase and in turn affect their overall quality of life [18]. Thus when they are not receiving the social support that they require this aggravates their already persisting condition into a worst state. The researcher during the interview also observed while interacting with the patients that when given help, guidance and freedom to engage into activities (for e.g. hobbies) of their choice they felt better and did not take their illness in a negative light as it's an end to their life and experience a sense of hopelessness. This was seen in the patients who were given the above freedom of expression as well love, care and understanding in one of the rehabilitation centres during data collection and thus they had low depression and high quality of life scores. Whereas those who got high scores on depression and low scores on quality of life were the patients who were moreover inpatients in the psychiatric residential centres or 
hospitals who were left by their family, which did not come to meet them or bothered staying in touch with them. They experienced more loneliness, more depression, low social support and quality of life.

During the interview process while interacting with the patients it was observed that even when such patients resided with their families not all families were understanding and considerate about their illness and state of mind. Some families did not help and support these patients, poor hygiene was maintained and they were restricted from indulging into any activities of their choice. Simple leisure activity of watching television was also restricted thus not allowing their freedom of choice and thus this would make them more vulnerable and feel rejected and thus leading to high chances of depression and poor quality of life. Our study had limitations in form that the data used for the analyses were collected through self-report instruments from individuals who were suffering from schizophrenia. Thus the state of mind of the participants had an effect on the results as some participants were suffering from chronic schizophrenia. A majority of the sample is collected from mental hospital and rehabilitation homes thus their disoriented state and low insight might have had an effect on the results.

Thus the study concludes that social support has an impact on depression and quality of life amongst the patients of schizophrenia. It's important to note gender differences are also present when studying the impact of social support on depression and quality of life among such patients. Thus by considering the influence of social support that has on such patients, its vital that they are given the best understanding, love, support and care to deal with this grave life long illness which had high comorbidity with depression and thus overall affects their quality of life. Thus the more awareness created about these factors along with the other pressing ones, it can bring about an overall positive impact on such patients and help them live their life with less feelings of hopelessness.

\section{REFERENCES}

1. Buchanan J. Social support and schizophrenia: a review of the literature. Arch Psych Nurs 1995;9:68-76.

2. Yanos PT, Moos RH. Determinants of functioning and well-being among individuals with schizophrenia: An integrated model. Clin Psychol Rev 2007;27(1):58-77.

3. Sawant SN, Jethwani KS. Understanding family functioning and social support in unremitting schizophrenia: A study in India. Indian J Psychiatry 2002;2:145-9.

4. Felce D, Perry J. Assessment of quality of life. Quality of Life. Conceptualization and Measurement. American Association on Mental Retardation (AAMR). 1996.

5. Bengtsson-Tops A, Hansson L. Quantitative and qualitative aspects of the social network in schizophrenic patients living in the community. Relationship to sociodemographic characteristics and clinical factors and subjective quality of life. Int J Soc Psychiatry 2001; 47(3):67-77.

6. Rudnick A, Kravetz S. The relation of social support-seeking to quality of life in schizophrenia. J Nerv Ment Disease 2001;189(4):258-62.

7. Corrigan PW, Phelan SM. Social support and recovery in people with serious mental illnesses. Comm Ment Health J 2004;40(6):513-23.

8. Malm U, May PR, Dencker SJ. Evaluation of the quality of life of the schizophrenic outpatient: a checklist. Schizophr Bull 1981;7(3):477-87.

9. Knights A, Hirsch SR. 'Revealed' depression and drug treatment for schizophrenia. Arch Gen Psychiatry 1981;38(7):806-11. 
10. Zimet GD, Dahlem NW, Zimet SG, Farley GK. The Multidimensional Scale of Perceived Social Support. J Personal Assess 1988;52:30-41.

11. Addington D, Addington J, Maticka-Tyndale E. Assessing depression in schizophrenia: the Calgary Depression Scale. Br J Psychiatry 1993.

12. The WHOQOL group: Development of World Health Organization Quality Of Life: rationale and current status.1992.

13. Beels CC. Schizophrenia and social support. Schizophr Bull 1981;1:58-72.

14. Hammer M. Social support, social network and schizophrenia. Schizophr Bull 1981;1:4557.

15. Tharayil D. Self, Social, and Family Perceptions in Relation to Loneliness Controlling for Depression among Individuals with Schizophrenia. The Internet Journal of Mental Health; $2006 ; 4(1)$.

16. Lin N, Woefel M, Light $S$. The buffering effects of social support subsequent to an important life event. J Health Soc Behav 1985;26:247-63.

17. McCorkle B, Tugers ES, Dunn EC, Layas A, Wan YM. Increasing social support for individuals with serious mental illness: Evaluating the compeer model of intentional friendship. Comm Ment Health J 2008;44(5):359-66.

18. Hafner H, Maurer K, Trendler G, Heiden W, Schmidt M, Konnecke R. Schizophrenia and depression: Challenging the paradigm of two separate diseases- a controlled study of schizophrenia, depression and healthy controls. Schizophr Res 2005;77(1):11-24.

Acknowledgements - Nil

Conflict of Interest - Nil

Funding - Nil. 